\title{
Regenerative Endodontics
}

\author{
Paul Roy Cooper* \\ Faculty of Dentistry, Sir John Walsh Research Institute, University of Otago, Dunedin, New Zealand
}

Keywords: pulp biology, pulp-dentin complex, stem cells, tissue regeneration, angiogenesis

\section{OPEN ACCESS}

Edited by:

Henry Fergus Duncan,

Trinity College Dublin, Ireland

Reviewed by:

Ariadne Letra,

University of Texas Health Science

Center at Houston, United States Matthias Widbiller,

University of Regensburg, Germany

*Correspondence:

Paul Roy Cooper p.cooper@otago.ac.nz

Specialty section:

This article was submitted to

Endodontics,

a section of the journal

Frontiers in Dental Medicine

Received: 19 July 2020

Accepted: 06 August 2020

Published: 08 September 2020

Citation:

Cooper PR (2020) Regenerative

Endodontics. Front. Dent. Med. 1:10.

doi: 10.3389/fdmed.2020.00010
A thorough understanding of basic and developmental biology in the dentine-pulp complex is essential to underpin therapeutic advancements in the field of regenerative endodontics. Improved knowledge of how dental and biomaterials interact with healthy and diseased tissue is required to enable improvements in minimally invasive vital pulp treatment (VPT). The tooth has an innate ability for repair following traumatic and carious injury and several processes come into play including the tissue's detection and resolution of infection and its inflammatory response, alongside the regulatory role that these processes play on tissue regenerative events, including tertiary dentinogenesis. The "repair" or tertiary dentine which can occur within the tooth in response to clinical treatments has been purposefully termed reactionary and reparative dentine. This enables focus on two different processes. Reactionary dentine is produced by surviving primary odontoblasts and occurs in response to less severe injury. Reparative dentinogenesis occurs due to more intense injury which results in the loss of the original developmentally derived odontoblasts, which are then replaced by new odontoblast-like cells which differentiate from progenitor/stem cells recruited to the injury site, either from the local tissue, or potentially from more distant niches. The tertiary dentine produced by the two processes is very different due to the diverse biological responses involved. Whilst molecular techniques are being utilized the complete characterization of the odontoblast-like cell phenotype remains elusive and the degree by which these cells mimic the original developmentally derived odontoblasts remains unclear. Nevertheless, the harnessing of the tertiary dentinogenic events alongside restorative procedures are clearly clinically important and advantageous. Whilst endodontic imaging approaches provide disease information, ideally to tailor clinical treatments, novel diagnostic approaches need to be developed to inform treatment planning. Currently, however, these approaches are subjective and therefore limited in their utility. Along with the difficulties associated with identifying suitable reproducible diagnostic biomarkers, it is also unclear as to which tissues or fluid sources are best to use which will then enable the optimal clinical approaches. Indeed, the clinical outcome which benefits the patient in terms of resolution of disease, absence of pain and swelling, restoration of tooth functionality and longevity, and tooth aesthetics to be applied are arguably the most important. It is therefore beneficial that clinician-scientist partnerships exist which enable meaningful studies and underpinning this is the need to ask the most important and critical research questions. Furthermore, due to the complex and multifaceted nature of the disease research in endodontics needs to encompass the role of the microbiology, the hosts immune and inflammatory response, stem cell and tissue regenerative biology, as well as the development of novel biomaterials which work to enhance dental tissue repair. Whilst animal models provide valuable information that can inform the development of novel endodontic treatments, it is the well-designed and targeted clinical trials that will be essential to show their efficacy and hence inform the uptake of these new clinical procedures. Regenerative endodontics should therefore embrace the repair, replacement 
and regeneration of the dentine-pulp complex. Research into this area is complex and requires multiple stake-holders which have suitable expertise to enable the clinical impact necessary for patient benefit.

\section{AUTHOR CONTRIBUTIONS}

The author confirms being the sole contributor of this work and has approved it for publication.
Conflict of Interest: The author declares that the research was conducted in the absence of any commercial or financial relationships that could be construed as a potential conflict of interest.

Copyright (c) 2020 Cooper. This is an open-access article distributed under the terms of the Creative Commons Attribution License (CC BY). The use, distribution or reproduction in other forums is permitted, provided the original author(s) and the copyright owner(s) are credited and that the original publication in this journal is cited, in accordance with accepted academic practice. No use, distribution or reproduction is permitted which does not comply with these terms. 